\title{
Successful kidney transplantation for a dialysis patient with anthracycline-induced cardiomyopathy: a case report
}

\author{
Suguru Yoneda $\cdot$ Masayoshi Okumi • \\ Seiji Yamaguchi
}

Received: 14 August 2012/Accepted: 15 October 2012/Published online: 7 November 2012

(C) Japanese Society of Nephrology 2012

Keywords Anthracycline-induced cardiomyopathy · Hemodialysis · Kidney transplantation · Uremic cardiomyopathy

Progress in the field of anticancer agent development has facilitated the long-term survival of children with malignancies. Anthracycline is a widely used anticancer agent that is well known to cause fatal cardiotoxicity, a condition termed anthracycline-induced cardiomyopathy (AIC). Routine use of angiotensin converting enzyme inhibitor (ACEi) is recommended as the first-line treatment for AIC. Although ACEi has a short-term beneficial effect on cardiac function [1], it cannot prevent the progression of cardiac dysfunction associated with AIC, and its long-term effectiveness is unclear [2]. Here we present a case of successful kidney transplantation that helped to prevent aggravation of left ventricular (LV) function in AIC.

A 22-year-old man was admitted because he complained of cardiac symptoms equivalent to NYHA III. He underwent systemic anticancer chemotherapies, including anthracycline, to treat Burkitt's lymphoma 17 years ago. Although the Burkitt's lymphoma was cured completely, he suffered from AIC and end-stage renal failure due to the adverse effects of the anticancer agents. He had started peritoneal dialysis and was treated with $\beta$-blocker and ACEi 12 years before. At the time of admission, an echocardiogram revealed a LV ejection fraction (LVEF) of 0.23 , severe diffuse LV hypokinesis, and an enlarged bilateral chamber. A coronary angiogram demonstrated

S. Yoneda $\cdot$ M. Okumi $(\bowtie) \cdot$ S. Yamaguchi

Department of Urology, Osaka General Medical Center,

3-1-56 Mandai-Higashi, Sumiyoshi, Osaka 558-8558, Japan

e-mail: okumi@uro.med.osaka-u.ac.jp normal coronary arteries and a heart biopsy revealed myocardial interstitial fibrosis compatible with druginduced cardiomyopathy (Fig. 1a). Appropriate hemodialysis management improved his cardiac symptoms and increased his LVEF to 0.48 . Because his cardiac function after hemodialysis was the same as that observed five years before, we believed that he would tolerate kidney transplantation surgery. Six months after admission, he underwent a living-related renal transplantation and produced urine soon after the transplantation. His LV function improved as his LVEF increased to 0.6, which has been maintained along with a favorable renal allograft function for four years (Fig. 1b).

Early-onset AIC, which occurs during the year after the completion of chemotherapy, is largely irreversible [3]. However, it has been reported that late-onset AIC can be reversed in almost two-thirds of cases [4]. The exact factors responsible for AIC irreversibility are unknown.

It is widely accepted that the heart and kidney are affected by each other, and chronic kidney disease may also cause heart failure in the absence of coronary artery disease. Uremic cardiomyopathy is expressed as LV dilatation and systolic dysfunction. Although the uremic toxins are not yet to be clearly determined, the uremic milieu can affect myocardial contractility and function. It has been shown that LV function improves after successful kidney transplantations [5]. We presume that the main reason for the improvement in LV function in our patient was recovery from the uremic milieu associated with AIC, and this recovery from the uremic state helped to stop the progression of AIC itself.

The present case indicates that kidney transplantation can be safely performed in a dialysis patient with AIC, and that successful kidney transplantation may even help to prevent aggravation of LV function. 
Fig. 1 a A heart biopsy revealed myocardial interstitial fibrosis compatible with druginduced cardiomyopathy. b Trends in echocardiography parameters such as left ventricular ejection fraction (LVEF), left ventricular fractional shortening (LVFS), left ventricular end-diastolic diameter (LVEDD), and left ventricular end-systolic diameter (LVESD) are shown from five years before kidney transplantation (Tx) until four years after $\mathrm{Tx}$ a

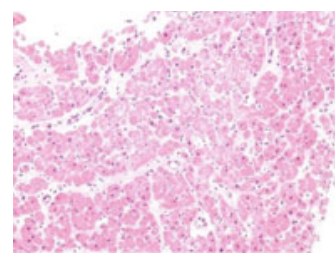

HE stain (x50)

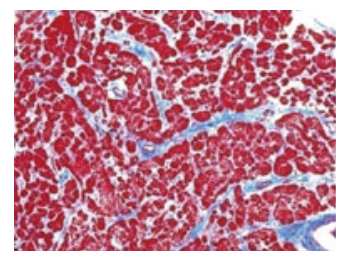

Azan-Mallory stain (x50)

b
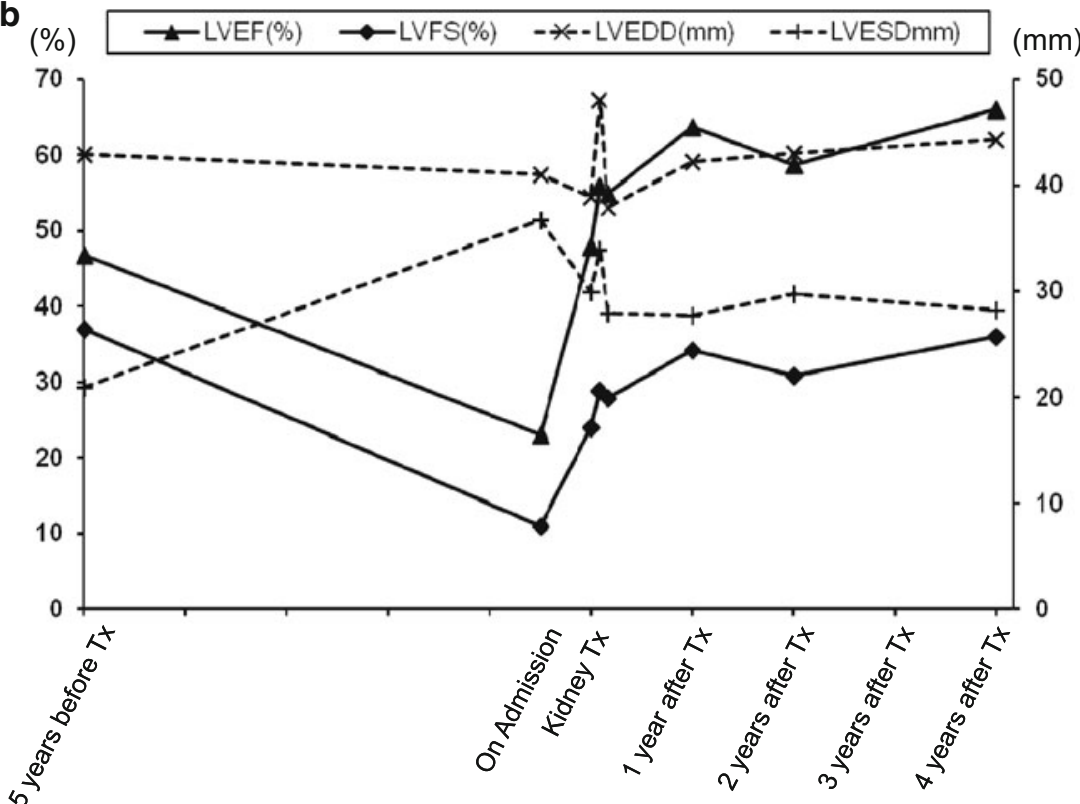

Conflict of interest The authors have declared that there is no conflict of interest.

\section{References}

1. Keefe DL. Anthracycline-induced cardiomyopathy. Semin Oncol. 2001;28:2-7.

2. Lipshultz SE, Colan SD. Cardiovascular trials in long-term survivors of childhood cancer. J Clin Oncol. 2004;22:769-73.
3. Haq MM, Legha SS, Choksi J, et al. Doxorubicin-induced congestive heart failure in adults. Cancer. 1985;56:1361-5.

4. Kumar S, Marfatia R, Tannenbaum S, Yang C, Avelar E. Doxorubicin-induced cardiomyopathy 17 years after chemotherapy. Tex Heart Inst J. 2012;39:424-7.

5. Wali RK, Wang GS, Gottlieb SS, et al. Effect of kidney transplantation on left ventricular systolic dysfunction and congestive heart failure in patients with end-stage renal disease. J Am Coll Cardiol. 2005;45:1051-60. 